\title{
Scattering analysis of 2DEG carrier extracted by QMSA in undoped $\mathrm{Al}_{0.25} \mathbf{G a}_{0.75} \mathrm{~N} / \mathrm{GaN}$ heterostructures
}

\author{
S B Lisesivdin ${ }^{1}$, S Acar ${ }^{1}$, M Kasap ${ }^{1}$, S Ozcelik ${ }^{1}$, S Gokden ${ }^{2}$ \\ and E Ozbay ${ }^{3}$ \\ ${ }^{1}$ Department of Physics, Gazi University, Ankara, Turkey \\ ${ }^{2}$ Department of Physics, Balikesir University, Balikesir, Turkey \\ ${ }^{3}$ Nanotechnology Research Center, Department of Physics, Department of Electrical-Electronics Engineering, Bilkent University, Ankara, \\ Turkey \\ E-mail: sblisesivdin@gmail.com
}

Received 10 December 2006, in final form 9 March 2007

Published 30 March 2007

Online at stacks.iop.org/SST/22/543

\begin{abstract}
Hall effect measurements on undoped $\mathrm{Al}_{0.25} \mathrm{Ga}_{0.75} \mathrm{~N} / \mathrm{GaN}$ heterostructures grown by a metalorganic chemical vapour deposition (MOCVD) technique have been carried out as a function of temperature (20-350 K) and magnetic field $(0-1.5 \mathrm{~T})$. Magnetic field dependent Hall data were analysed using the quantitative mobility spectrum analysis (QMSA) technique. The mobility and density within the two-dimensional electron gas (2DEG) at the $\mathrm{Al}_{0.25} \mathrm{Ga}_{0.75} \mathrm{~N} / \mathrm{GaN}$ interface and within the underlying $\mathrm{GaN}$ layer were successfully separated by QMSA. Mobility analysis has been carried out using both the measured Hall data at a single field and the extracted data from QMSA. Analysis of the temperature-dependent mobility of 2DEG extracted from QMSA indicates that the interface roughness and alloy disorder scattering mechanisms are the dominant scattering mechanisms at low temperatures while at high temperatures only polar optical phonon scattering is the dominant mechanism. $\mathrm{Al}_{0.25} \mathrm{Ga}_{0.75} \mathrm{~N} / \mathrm{GaN}$ interface related parameters such as well width, deformation potential constant and correlation length were also accurately obtained from the fits of the simple analytical expressions of scattering mechanisms to the 2DEG mobility.
\end{abstract}

\section{Introduction}

High electron mobility transistors (HEMTs) are widely used and accepted as the promising components of the high-speed electronics. HEMTs based on $\mathrm{Al}_{x} \mathrm{Ga}_{1-x} \mathrm{~N} / \mathrm{GaN}$ heterostructures are the most interesting candidate since their description in 1993 [1] and demonstration of high-power operability [2]. Due to their large bandgap energy, large electron drift velocities, high conduction band discontinuity and high thermal stability, $\mathrm{Al}_{x} \mathrm{Ga}_{1-x} \mathrm{~N} / \mathrm{GaN}$ heterostructures can operate at high power and high temperature conditions with a 2DEG density and high mobility values as compared even with GaAs-based devices [3-6]. Even without an intentional doping, due to strong spontaneous and piezoelectric polarizations at the $\mathrm{Al}_{x} \mathrm{Ga}_{1-x} \mathrm{~N} / \mathrm{GaN}$ interface, $\mathrm{Al}_{x} \mathrm{Ga}_{1-x} \mathrm{~N} / \mathrm{GaN}$ heterostructures have 2DEG density with high sheet carrier density values [7, 8]. Since the mobility and density of the 2DEG in heterostructures are the key parameters related to the device performance, a detailed study of these parameters together with scattering mechanisms is of 
critical importance for the device applications of $\mathrm{Al}_{x} \mathrm{Ga}_{1-x} \mathrm{~N} / \mathrm{GaN}$ heterostructures. The analyses of carrier transport properties in $\mathrm{Al}_{x} \mathrm{Ga}_{1-x} \mathrm{~N} / \mathrm{GaN}$ heterostructures have been reported in a number of papers [9-12]. In the majority of cases, these studies have been based on the analysis of the temperature dependent single magnetic field Hall measurements. In the mixed conduction (multi carriers) case, the standard resistivity and Hall effect measurements at a single magnetic field are of limited use because these measurements provide only averaged values of mobility and carrier density. Therefore,

Table 1. Material constants used in scattering calculations [20, 32].

\begin{tabular}{ll}
\hline High frequency dielectric constant & $\varepsilon_{\infty}=5.35 \varepsilon_{\mathrm{s}}=$ \\
Static dielectric constant & $8.9 \mathrm{~m}^{*}=$ \\
Electron effective mass & $0.22 \mathrm{~m}_{0}$ \\
$\begin{array}{l}\text { LO-phonon energy LA- } \\
\text { phonon velocity }\end{array}$ & $\mathrm{h} \omega^{-}=0.092 \mathrm{eV}$ \\
$\begin{array}{l}\text { Density of crystal } \\
\text { Electron wave vector }\end{array}$ & $\mathrm{u}_{\mathrm{l}}=6.56 \times 10^{3} \mathrm{~m} \mathrm{~s}^{-1} \rho=$ \\
$\begin{array}{l}\text { The electromechanical coupling } \\
\text { coefficient }\end{array}$ & $6.15 \times 10^{3} \mathrm{~kg} \mathrm{~m}^{-3} \mathrm{k}=7.3$ \\
& $\times 10^{8} \mathrm{~m}^{-1}$ \\
& $\mathrm{~K}^{2}=0.039$ \\
LA elastic constant & $\mathrm{c}_{\mathrm{LA}}=2.650 \times 10^{11} \mathrm{~N} \mathrm{~m}^{-2} \mathrm{c}_{\mathrm{TA}}$ \\
TA elastic constant & $=0.442 \times 10^{11} \mathrm{~N} \mathrm{~m}^{-2} \mathrm{x}=$ \\
$\begin{array}{l}\text { Alloy mole fraction } \\
\text { Lattice constant in the }(0001)\end{array}$ & $0.25 \mathrm{c}=5.185 \times 10^{-10} \mathrm{~m}$ \\
$\quad$ direction & $\Omega_{0}=3.48487 \times 10^{-29} \mathrm{~m}^{-3}$ \\
Volume of one atom & $\mathrm{U}_{\mathrm{AL}}=2.36 \times 10^{-19} \mathrm{~V}$ \\
Alloy potential &
\end{tabular}

single magnetic field Hall characterization is incapable of providing precise determination of the transport properties of $\mathrm{Al}_{x} \mathrm{Ga}_{1-x} \mathrm{~N} / \mathrm{GaN}$ heterostructures since the charge carriers in bulk $\mathrm{GaN}$ and $\mathrm{Al}_{x} \mathrm{Ga}_{1-x} \mathrm{~N}$ layer can also contribute to the measurementsinadditiontotheinduced2DEGbypolarization at the interface.

To extract the correct transport parameters of the individualcarriersinthemultilayeredsemiconductormaterials such as $\mathrm{Al}_{x} \mathrm{Ga}_{1-x} \mathrm{~N} / \mathrm{GaN}$ heterostructures, resistivity and Hall effect measurements are to be performed as a function of magnetic field. These measurements (variable field) allow the densities and mobilities to be simultaneously characterized for each of the multiple carrier species. Recently, the magnetic field dependent Hall data have been analysed successfully using QMSA technique, which is an effective technique for determining individual carriers in the multilayered semiconductors [13, 14]. Using the QMSA technique, the mobilities and carrier densities of each electron and hole species in bulk InN and GaN epilayers, and InP/InAlGaAs, $\mathrm{HgCdTe}, \mathrm{AlGaAs} / \mathrm{GaAs}$ and $\mathrm{AlGaN} / \mathrm{GaN}$ heterostructures were investigated successfully by several groups [15-19].

In this work, firstly resistivity and Hall effect measurements of $\mathrm{Al}_{0.25} \mathrm{Ga}_{0.75} \mathrm{~N} / \mathrm{GaN}$ heterostructures grown by MOCVD technique have been carried out as a function of temperature $(20-350 \mathrm{~K})$ and magnetic field $(0-1.5 \mathrm{~T})$. To extract the individual mobilities and carrier densities of $\mathrm{Al}_{0.25} \mathrm{Ga}_{0.75} \mathrm{~N} / \mathrm{GaN}$ heterostructures, the measurement results were analysed using QMSA. Secondly, 2DEG mobility analyses by taking into consideration the most relevant scattering mechanisms are carried out using both the measured Hall data and the extracted 2DEG data from QMSA. In both cases, $\mathrm{Al}_{0.25} \mathrm{Ga}_{0.75} \mathrm{~N} / \mathrm{GaN}$ interface related parameters were obtained and the differences between two cases were also discussed.

\section{Scattering mechanisms}

Scattering mechanisms of two-dimensional (2D) carriers in III-V heterojunctions are well described ([20], see references therein). The analytical expressions of the scattering mechanisms used in our calculations are summarized below. The material parameters used in the calculations are also listed in table 1.

\subsection{Polar optical phonon scattering}

At high temperatures, the mobility of a 2D carrier is mostly limited by polar optic phonon scattering. The expression of the mobility limited by the polar optic phonon scattering is given by Ridley as [21]

$$
\mu_{\mathrm{po}}=\frac{4 \pi \varepsilon_{0} \varepsilon_{\mathrm{p}} \hbar^{2}}{e \omega m^{* 2} Z_{0}}\left[\mathrm{e}^{\hbar \omega / k_{\mathrm{B}} T}-1\right],
$$

where

$$
\frac{1}{\varepsilon_{\mathrm{p}}}=\frac{1}{\varepsilon_{\infty}}-\frac{1}{\varepsilon_{\mathrm{s}}}
$$

Here, ${ }^{-} \mathrm{h} \omega$ is the polar optic phonon energy, $\varepsilon_{\infty}$ and $\varepsilon_{\mathrm{s}}$ are the high and low frequency dielectric constants, respectively. $\mathrm{m}^{*}$ is the effective mass.

\subsection{Acoustic phonon scattering}

At intermediate temperatures, the acoustic phonon scattering is another important scattering mechanism. In this work, we use the elastic acoustic phonon scattering model proposed by Ridley et al [22]. The acoustic phonon scattering is calculated by considering two scattering mechanisms, including deformation potential and piezoelectric scattering. The mobility expression of deformation potential is given by [22]

$$
\mu_{\mathrm{dp}}=\frac{16 \rho e u_{1}^{2} \hbar^{3}}{3 \Xi^{2} k_{\mathrm{B}} T m^{* 2} b} \frac{1}{J_{\mathrm{DP}}(k)}
$$

In equation (3), $\rho$ is the crystal density, $u_{1}$ is the longitudinalacousticphononvelocityandisthedeformation potential. The factor $b$ is called the Fang-Howard expression [23] of wavefunctions for Hartree approximation of a triangular well and is given by [24]

$$
b=\left(\frac{33 e^{2} m^{*} n_{2 \mathrm{D}}}{8 \varepsilon_{0} \varepsilon_{\mathrm{s}} \hbar^{2}}\right)^{1 / 3} .
$$

In equation (3), $\mathrm{J}_{\mathrm{DP}}(\mathrm{k})$ is the integral

$$
J_{\mathrm{DP}}(k)=\int_{0}^{2 k} \frac{1}{2 \pi k^{3}\left(q+q_{\mathrm{s}}\right)^{2} \sqrt{1-\left(\frac{q}{2 k}\right)^{2}}} q^{4} \mathrm{~d} q .
$$

In this integral, $\mathrm{q}_{\mathrm{s}}$ is the two-dimensional reciprocal screening length which is defined as

$$
q_{\mathrm{s}}=\frac{e^{2} m^{*}}{2 \pi \hbar^{2} \varepsilon_{0} \varepsilon_{\mathrm{s}}} F_{11}(q) f(0) .
$$

Here, $f(0)$ is the occupation probability at the subband edge, which can be assumed that all screening is determined 
by the lowest subband electrons [22]. $\mathrm{F}_{11}(\mathrm{q})$ is the form factor of the ground-state Fang-Howard wavefunction [23].

In strongly polar materials such as $\mathrm{GaN}$ the acoustic phonons are strongly interacted by the piezoelectric effect. The mobility expression of piezoelectric scattering with a simplification of angular dependence is given by [22]

$$
\mu_{\mathrm{pe}}=\frac{\pi \varepsilon_{0} \varepsilon_{\mathrm{s}} \mathrm{h}^{-{ }^{3} \mathrm{k}} 1}{\mathrm{eK} 2 \mathrm{kB} \mathrm{Tm}_{* 2} \mathrm{JPE}(\mathrm{k}) .}
$$

In equation (7), $K$ is the electromechanical coupling coefficient and given by [25]

$$
\text { (8) } \varepsilon_{\mathrm{s}} c_{\mathrm{LA}}^{\mathrm{LA}}-{ }_{\varepsilon_{\mathrm{s}} c} K_{\mathrm{TA}}^{2}=\frac{\varepsilon^{2}}{\varepsilon_{\mathrm{TA}}^{2}} \text {. }
$$

In equation (8), $\varepsilon_{\mathrm{LA}}, \varepsilon_{\mathrm{TA}}, \mathrm{c}_{\mathrm{LA}}, \mathrm{c}_{\mathrm{TA}}$ are the effective piezoelectric constants and the averaged elastic constants related to longitudinal and transverse acoustic phonons, respectively. The integral $\mathrm{J}_{\mathrm{PE}}(\mathrm{k})$ is in the form

$$
J_{\mathrm{PE}}(k)=\int_{0}^{2 k} \frac{F_{11}(q)}{4 k^{2}\left(q+q_{\mathrm{s}}\right)^{2} \sqrt{1-\left(\frac{q}{2 k}\right)^{2}}} q^{3} \mathrm{~d} q .
$$

\subsection{Alloy disorder scattering}

It is well known that the investigated 2D carriers populate near the $\mathrm{Al}_{x} \mathrm{Ga}_{1-x} \mathrm{~N}$ layer. The scattering of these electrons by conduction band disorder is called alloy disorder scattering. Kearley and Horrell [26] gave the mobility expression of alloy disorder scattering without screening effects as

$$
\begin{aligned}
\mu_{\text {alloy }}= & \begin{array}{l}
16 \quad \mathrm{eh}^{-3} \\
3 b x(1-x) m^{* 2} \Omega_{0} U \\
2,(10)
\end{array}
\end{aligned}
$$

where $x$ is the alloy mole fraction, ${ }_{0}$ is the volume occupied by one atom and $\mathrm{U}_{\mathrm{AL}}$ is the alloy potential.

\subsection{Background impurity scattering}

Impurity scatterings for 2DEG carriers can be investigated in two parts; an ionized impurity scattering due to remote donors which is effective in modulation-doped structures and an ionized impurity scattering due to interface charges or simply background impurity scattering which is effective in all structures. In this work, because our samples are nominally undoped, we only use the background impurity scattering as an impurity scattering. The mobility of background impurity scattering is given by [27]

$$
\mu_{\mathrm{BI}}=\frac{8 \pi \hbar^{3} \varepsilon^{2} k_{\mathrm{F}}^{2} I_{\mathrm{B}}(\beta)}{\mathrm{e} 3 \mathrm{~m} * 2 \mathrm{NBI}},
$$

where $\varepsilon$ is the dielectric permittivity of $\mathrm{GaN}$, and $\mathrm{N}_{\mathrm{BI}}$ is the $2 \mathrm{D}$ impurity density due to background impurities. The integral $\mathrm{I}_{\mathrm{B}}(\beta)$ is defined as

$$
I_{\mathrm{B}}(\beta)=\int_{0}^{\pi} \frac{\sin ^{2} \theta}{(\sin \theta+\beta)^{2}} \mathrm{~d} \theta
$$

where

$$
\beta=\mathrm{S}_{0} / 2 \mathrm{k}_{\mathrm{F}}
$$

In equation (13), $\mathrm{k}_{\mathrm{F}}$ is the wavevector on the Fermi surface, and $\mathrm{S}_{0}$ is the screening constant which is given for the degenerate case by Lee et al as [28]

$$
S_{0}=\frac{e^{2} m^{*}}{2 \pi \varepsilon \hbar^{2}} .
$$

\subsection{Interface roughness scattering}

Interfaceroughnessisanimportantproblemforsemiconductor heterostructures [20]. Interface roughness can lead to the perturbation of electron energy [29]. Narrow quantum wells of $\mathrm{Al}_{x} \mathrm{Ga}_{1-x} \mathrm{~N} / \mathrm{GaN}$ heterostructures are more sensitive to interface roughness that can lead to a large fluctuation in the

cattering analysis of 2DEG extracted by QMSA in undoped $\mathrm{Al}_{0.25} \mathrm{Ga}_{0.75} \mathrm{~N} / \mathrm{GaN}$ quantized electron energies [30]. The mobility of interface roughness scattering is given by [20]

$$
\mu_{\mathrm{IFR}}=\left(\begin{array}{c}
2 \varepsilon_{0} \varepsilon_{\mathrm{S}} \\
n_{2 \mathrm{D}} \Delta \Lambda
\end{array}\right)^{2} \hbar^{3} e^{3} m^{2} J
$$

$\operatorname{IFR}(\mathrm{k})$

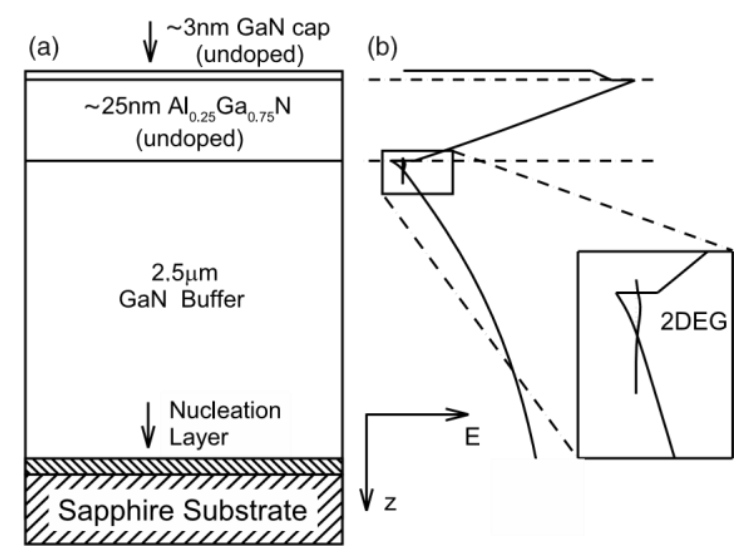

Figure 1. (a) Layer structure of $\mathrm{Al}_{0.25} \mathrm{Ga}_{0.75} \mathrm{~N} / \mathrm{GaN}$ heterostructures sample that used in study. (b) Band structure of the sample. 2DEG is shown in detail in the inlet.

where is the lateral size of the roughness and $\Lambda$ is the correlation length between fluctuations. The integral $\mathrm{J}_{\mathrm{IFR}}(\mathrm{k})$ in equation (15) is defined as

$$
J_{\text {IFR }}(k)=\int_{0}^{2 k} \frac{\mathrm{e}^{-q^{2} \Lambda^{2} / 4}}{2 k^{3}\left(q+q_{\mathrm{s}}\right)^{2} \sqrt{1-\left(\frac{q}{2 k}\right)^{2}}} q^{4} \mathrm{~d} q,
$$

where $\mathrm{q}_{\mathrm{s}}$ is the screening constant and given by [30]

$$
q_{\mathrm{s}}=\frac{e^{2} m^{*}}{2 \pi \hbar^{2} \varepsilon_{0} \varepsilon_{\mathrm{s}}} F(q) .
$$

The form factor $\mathrm{F}(\mathrm{q})$ in equation (17) is given by [31]

$$
F(q)=\int_{0}^{\infty} \mathrm{d} z \int_{0}^{\infty} \mathrm{d} z^{\prime}[f(z)]^{2}\left[f\left(z^{\prime}\right)\right]^{2} \mathrm{e}^{-q\left|z-z^{\prime}\right|} .
$$




\section{Experimental techniques}

The sample investigated in this work was grown on a c-plane (0001) sapphire $\left(\mathrm{Al}_{2} \mathrm{O}_{3}\right)$ substrate in a low-pressure MOCVD reactor. Prior to epilayer growth, the sapphire substrate was cleaned in $\mathrm{H}_{2}$ ambient at $1100{ }^{\circ} \mathrm{C}$, and then a $25 \mathrm{~nm}$ thick low temperature (LT) $\mathrm{GaN}$ nucleation layer was grown at $500{ }^{\circ} \mathrm{C}$. The reactor pressure was set to 50 mbar during the substrate cleaning and nucleation growth. After the deposition of the LT-GaN nucleation layer, the wafers were heated to a high temperature for annealing. For the sample, the two-step growth process was applied with the ramp time $2.5 \mathrm{~min}$, and the annealing temperature $1100{ }^{\circ} \mathrm{C}$. Approximately, a $2.5 \mu \mathrm{m}$ thick GaN layer was deposited on the annealed nucleation layers using constant growth conditions. Finally, a $25 \mathrm{~nm}$ thick $\mathrm{Al}_{0.25} \mathrm{Ga}_{0.75} \mathrm{~N}$ with $3 \mathrm{~nm} \mathrm{GaN}$ cap layers was grown. All layers are nominally undoped. The details of the samples are given in figure 1.

For the resistivity and Hall effect measurements by the van der Pauw method, square-shaped $\left(5 \times 5 \mathrm{~mm}^{2}\right)$ samples havebeenpreparedwithTi/Al/Ni/Auevaporateddotcontacts in the corners. Current was kept low to maintain ohmic behaviour, so the $2 \mathrm{D}$ electrons were in thermal equilibrium with the lattice. Current independence of mobility and the carrier density has been confirmed in the current interval of $1-500 \mu \mathrm{A}$. The measurements have been made over a

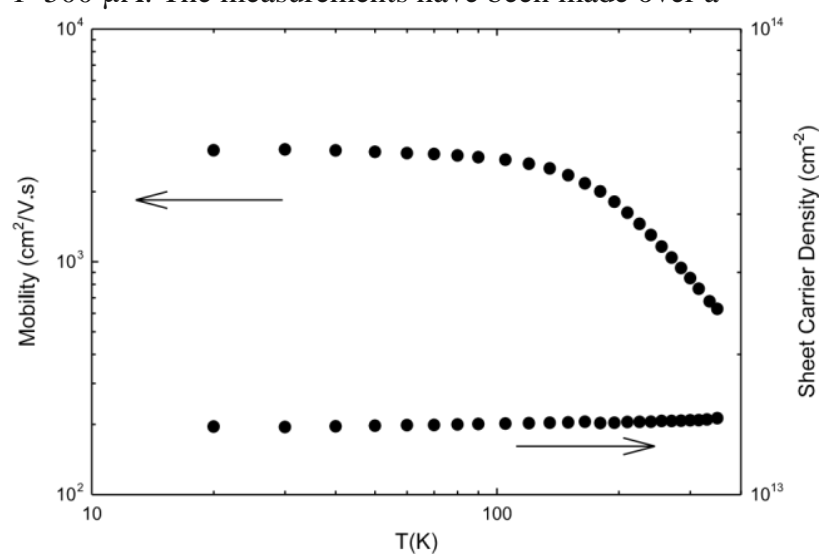

Figure 2. Experimental Hall mobility and sheet carrier density in undoped $\mathrm{Al}_{0.25} \mathrm{Ga}_{0.75} \mathrm{~N} / \mathrm{GaN}$.

temperature range 20-350 K using a Lakeshore Hall effect measurement system (HMS). At each temperature step, the Hall coefficient and resistivity have been measured for both current directions, both magnetic field polarization, and all possible contact configurations at 31 magnetic field steps between 0 and $1.5 \mathrm{~T}$. The magnetic field dependent data have been analysed using QMSA software provided by Lakeshore.

\section{Experimental results and analysis}

4.1. Experimental results and quantitative mobilityspectrum analysis

Resistivity and Hall effect measurements of $\mathrm{Al}_{0.25} \mathrm{Ga}_{0.75} \mathrm{~N} / \mathrm{GaN}$ heterostructures have been carried out as a function of temperature $(20-350 \mathrm{~K})$ and magnetic field (0-1.5 T). Figure 2 shows the temperature dependence of the sheet carrier density and Hall mobility at $0.5 \mathrm{~T}$ in the temperature range of 20-350 K. At high temperatures, the mobility sharply decreases with increasing temperature while it is independent of temperature at low temperatures (below $100 \mathrm{~K}$ ). The sheet carrier density can be accepted as temperature independent. These behaviours of sheet carrier density and mobility are typical of 2DEG structures. Above $100 \mathrm{~K}$, Hall mobility decreases with increasing temperature with a temperature dependence of $\sim \mathrm{T}^{-3 / 2}$, which is a typical temperature dependence of phonon scattering mobility. The sheet carrier density still tends to be a constant, which is a further confirmation of the existence of 2DEG even at high temperatures. At room temperature, Hall mobility and sheet carrier density of the sample are $850 \mathrm{~cm}^{2} \mathrm{~V}^{-1} \mathrm{~s}^{-1}$ and $1.44 \times$ $10^{13} \mathrm{~cm}^{-2}$, respectively. At $20 \mathrm{~K}$, electron mobility is as high as $3013 \mathrm{~cm}^{2} \mathrm{~V}^{-1} \mathrm{~s}^{-1}$. In the nominally undoped $\mathrm{Al}_{x} \mathrm{Ga}_{1-x} \mathrm{~N} / \mathrm{GaN}$ heterostructures, such a high value of sheet carrier densities is due to the spontaneous and piezoelectric polarization fields $[33,34]$.

Magnetic field dependent Hall data taken at a temperature range of 20-350 K are analysed by using QMSA technique. A

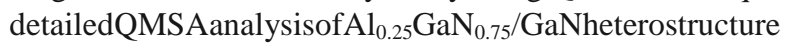
wasgiveninourpreviouswork[35]. Figures3(a)and(b)show the QMSA results as a function of temperature for the mobility and the integrated density for the studied sample. From
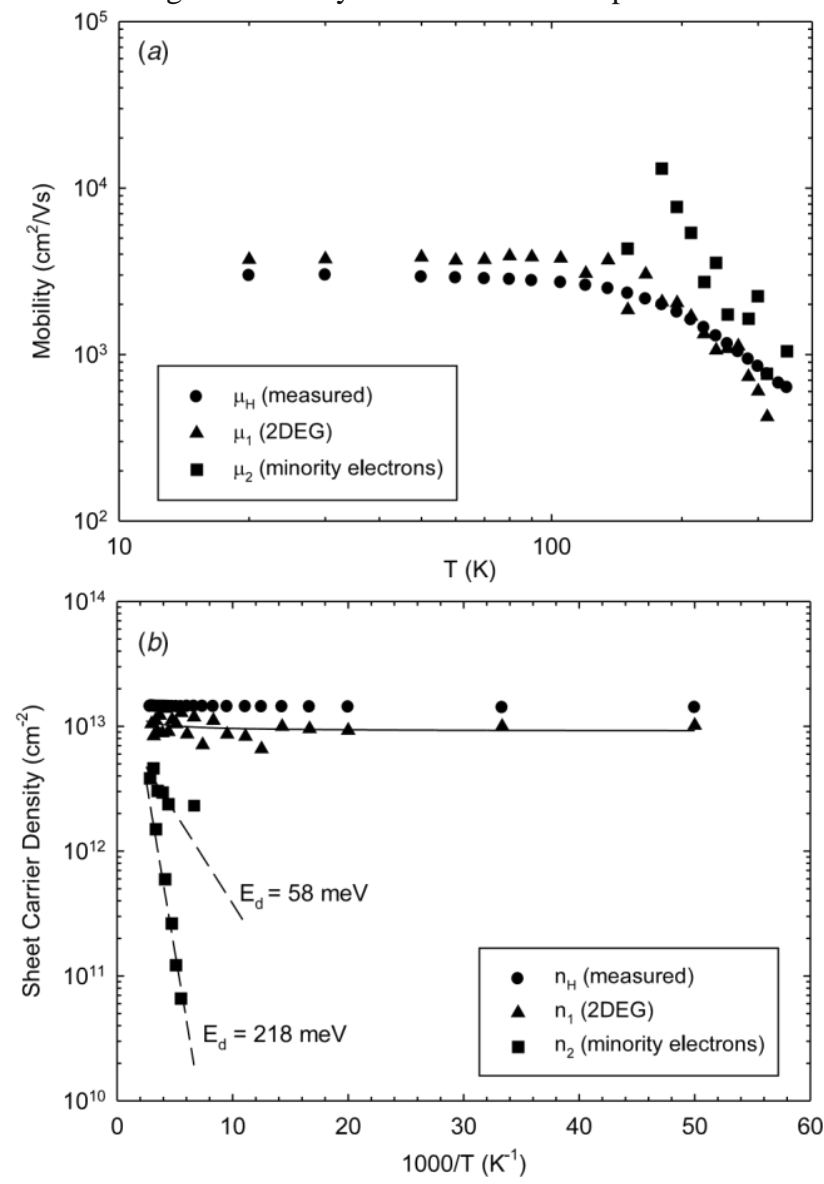

Figure 3. (a) Mobility versus temperature. The circle represents measured mobility. The triangle and square represent mobilities obtained from QMSA. (b) Sheet carrier density versus inverse temperature. The circle represents measured sheet density. The triangle and square represent the $2 \mathrm{DEG}$ and minority carrier 
densities obtained from QMSA, respectively. Lines show the proposed trends of the $2 \mathrm{DEG}$ and minority carriers.

figure $3(b)$, it can be clearly understood that both polarizationinduced 2DEG density (denoted with triangles) and thermally activated carriers (denoted with squares) contribute to the measured carrier density. At low temperatures, the extracted 2DEG mobility is slightly higher than the measured mobility. Below $100 \mathrm{~K}$, the 2DEG mobility is temperature independent. Above $100 \mathrm{~K}$, the 2DEG mobility is limited by lattice scattering mechanisms, which will be analysed in the next section. The extracted 2DEG density is independent of temperature at the whole studied temperature range, as is expected. Its proposed trend with temperature is shown by the solid line in figure $3(b)$. On the other hand, at the whole studied temperature range, the extracted 2DEG density is also lower than the measured sheet carrier density. The mobilities and densities of the minority carriers are highly temperature dependent. The thermally activated minority carriers (with activation energies of 58 and $218 \mathrm{meV}$ ) are originated from the donor levels of bulk GaN $[13,35]$.

\subsection{Mobility analysis}

In this section the mobilities limited by the individual scatteringmechanisms, polaropticalphonon,acousticphonon,

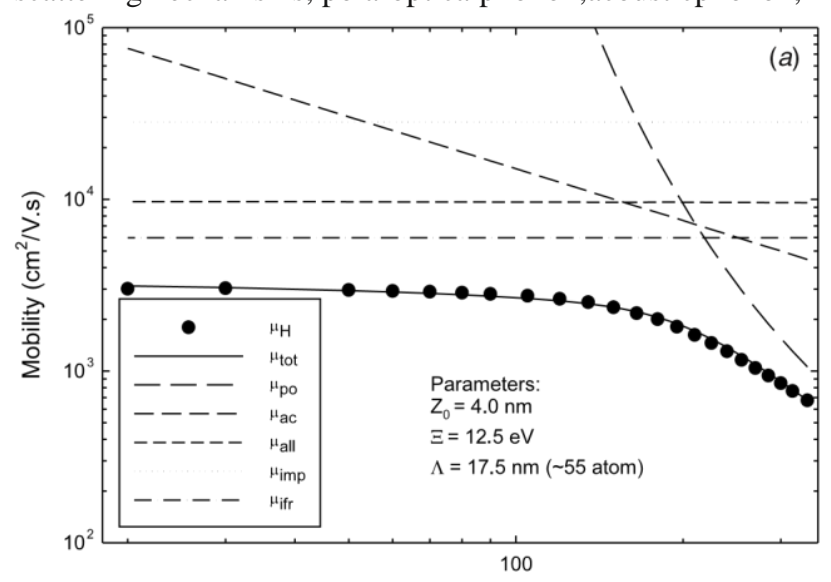

$\mathrm{T}(\mathrm{K})$

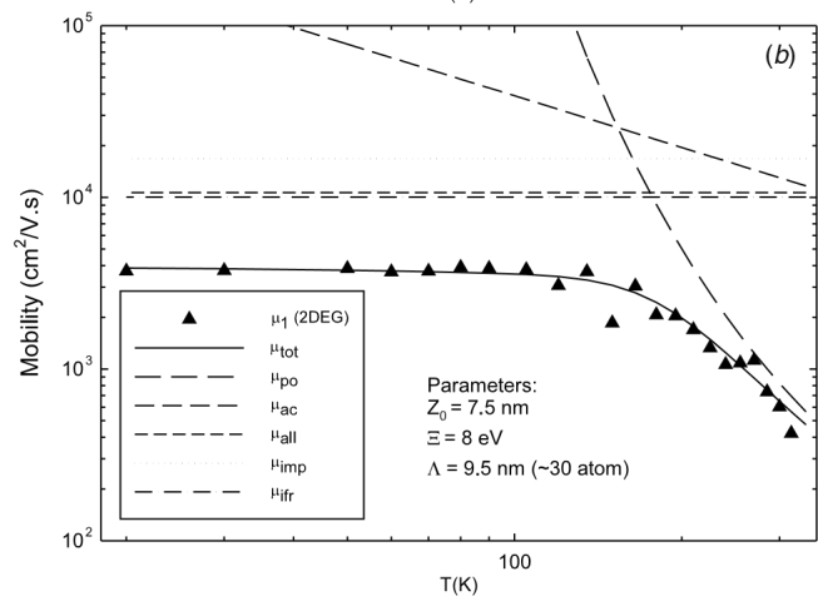

Figure 4. (a) Measured and calculated (using the measured Hall data) mobility versus temperature. (b) Extracted and calculated (using the extracted 2DEG density and mobility) mobility versus temperature. alloy disorder, background impurity and interface roughness scatterings have been calculated from the expressions given in section 2 using the material parameters given in table 1 . In the calculation, the background impurity $\left(n_{\text {imp }}\right)$ and lateral size ( ) were taken as $10^{-23} \mathrm{~m}^{-3}[36]$ and $2 \times 2.58 \times 10^{-10} \mathrm{~m}$

(for two monolayers) [32], respectively. The other parameters such as well width $\left(Z_{0}\right)$, deformation potential constant () and correlation length ( ) were used as adjustable parameters. Using the Mattheisen rule, the total mobility is then calculated as the combination of individual mobilities.

Firstly, we carried out the fit of the scattering expressions to the experimental mobility using the measured sheet carrier density taken at $0.5 \mathrm{~T}$, as the usual approach. The calculated individual mobilities and the total mobility are given in figure $4(a)$. It can be seen from figure 4(a) that the total mobility fits quite well to the experimental data, taken at $0.5 \mathrm{~T}$, using a well width of $Z_{0}=4 \mathrm{~nm}$, a deformation potential constant of $\Xi=$ $12.5 \mathrm{eV}$ and a correlation length of $=17.5 \mathrm{~nm}$ (corresponds to approximately 55 atomic spacing).

In general, the single field Hall effect data are widely used in the scattering analysis. However, since the single field Hall effect measurements provide only a weighted average of the mobility and carrier density, an accurate scattering analysis can only be carried out in the case of single carrier conduction. If the structure contains multiple carriers such as in $\mathrm{Al}_{x} \mathrm{Ga}_{1-x} \mathrm{~N} / \mathrm{GaN}$ heterostructures, the single field Hall effect measurements would not lead to identify the correct transport parameters of the individual carriers. Secondly, the above calculations were, therefore, repeated using the extracted 2DEG mobility and 2DEG density from QMSA. The results are given in figure 4(b). A good agreement with the extracted 2DEG mobility is obtained using the fitting parameters as the well width of $Z_{0}=7.5 \mathrm{~nm}$, the deformation potential constant of $\Xi=8 \mathrm{eV}$ and the correlation length of $\Lambda=9.5 \mathrm{~nm}$ (corresponds to approximately 30 atomic spacing).

The fitting parameters of the 2DEG obtained from the scattering analysis using the data extracted from QMSA show significantdifferencesfromthatoftheobtainedfromthesingle field Hall data. The former analysis has a smaller well width, a higher deformation potential value and a higher correlation length. Since the second analysis has been based on only the 2DEG mobility and density without the effects of the other carriers, the calculated parameters can be considered as more accurateparametersrepresentingtherealsamplefigures. Itcan be seen from figures $4(a)$ and $(b)$ that the values of calculated individual mobility are also considerably different than that of the obtained from the single field Hall data. This indicates thatanyconclusiondrawnfromsinglefieldHallmeasurements may be highly misleading.

Considering the results (according to figure 4(b)), we conclude: (i) the low-temperature mobility is dominated by interface roughness and alloy disorder scattering mechanisms, and the background impurity scattering has also a

Scattering analysis of $2 \mathrm{DEG}$ extracted by QMSA in undoped $\mathrm{Al}_{0.25} \mathrm{Ga}_{0.75} \mathrm{~N} / \mathrm{GaN}$ considerable influence on the 2DEG formed at the interface in undoped $\mathrm{Al}_{0.25} \mathrm{Ga}_{0.75} \mathrm{~N} / \mathrm{GaN}$ heterostructures. (ii) At high temperatures, the optical phonon scattering is the dominant mechanism, and the effects of interface roughness and alloy 
disorder scattering mechanisms have only small contribution to the mobility of 2DEG. It is interesting to note that our analyses show the acoustic phonon scattering has a negligible effect on the 2DEG mobility. However, the acoustic phonon scattering mechanism is, in general, found as a main scattering mechanism at intermediate temperature [37-39]. (iii) For $\mathrm{Al}_{0.25} \mathrm{Ga}_{0.75} \mathrm{~N} / \mathrm{GaN}$ heterostructures, the well width of $Z_{0}=7.5$ $\mathrm{nm}$, the deformation potential constant of $\Xi=8 \mathrm{eV}$ and the correlation length of $\Lambda=9.5 \mathrm{~nm}$ are obtained. These values are superior to the single-field measurement analysis results when compared with the Fermi wavelength well-width approximation [40] and the Hsu et al calculation for the deformation potential [9].

\section{Conclusions}

Hall effect measurements on undoped $\mathrm{Al}_{0.25} \mathrm{Ga}_{0.75} \mathrm{~N} / \mathrm{GaN}$ heterostructures grown by MOCVD have been carried out as a function of temperature $(20-350 \mathrm{~K})$ and magnetic field (0$1.5 \mathrm{~T}$ ). Magnetic field dependent Hall data were analysed using QMSA technique. QMSA successfully separated electrons in the $2 \mathrm{DEG}$ at the $\mathrm{Al}_{0.25} \mathrm{Ga}_{0.75} \mathrm{~N} / \mathrm{GaN}$ interface and electrons in the bulk GaN layer which contribute to the measurements in addition to the 2DEG at high temperatures. The mobility analysis has been carried out both using the measured Hall data at a single field $(B=0.5 \mathrm{~T})$ and the data extracted from QMSA. The scattering analysis based on the extracted mobility and density of 2DEG formed at an $\mathrm{Al}_{0.25} \mathrm{Ga}_{0.75} \mathrm{~N} / \mathrm{GaN}$ heterointerface shows that the interface roughness and alloy disorder scattering mechanisms are the dominant scattering mechanisms at low temperatures while at high temperatures only the polar optical phonon scattering is the dominant mechanism. The values of well width, deformation potential and correlation length were found as 7.5 $\mathrm{nm}, 8 \mathrm{eV}$ and $9.5 \mathrm{~nm}$, respectively. Finally, it can be concluded that any conclusion drawn from single field Hall data or from any analysis based on single field Hall measurements would be highly misleading not only for $\mathrm{Al}_{x} \mathrm{Ga}_{1-x} \mathrm{~N} / \mathrm{GaN}$ heterostructures but also for any semiconductor structures with multicarrier conduction.

\section{Acknowledgments}

This work is supported by the State of Planning Organization of Turkey under grant no. 2001K120590, and by TUBITAK under project nos. 104E090, 105E066, 105A005. One of the authors (Ekmel Ozbay) acknowledges partial support from the Turkish Academy of Sciences.

\section{References}

[1] Khan M A, Bhattarai A, Kuznia J N and Olson D T 1993 Appl. Phys. Lett. 631214

[2] Wu Y-F, Keller B P, Keller S, Kapolnek D, Kozodoy P, Denbaars S P and Mishra U K 1996 IEEE Electron Device Lett. 17455

[3] Wu Y-F, Keller B P, Parikh P, Kapolnek D, Denbaars S P and Mishra U K 1997 IEEE Electron Device Lett. 18290

[4] Gaska R, Chen Q, Yang J, Osinsky A, Khan M A and Shur M S 1997 IEEE Electron Device Lett. 18492 [5] Ruden P P, Albrecht J D, Sutandi A, Binari S C, Anastasiou K I, Ancona
M G, Henry R L, Koleske D D and Wickenden A E 1999

MRS Internet J. Nitride Semicond.

Res. 4S1 G6.35

[6] Sheppard S T, Doverspike K, Pribble W L, Allen S T, Palmour J W, Kehias L T and Jenkins T J 1999 IEEE Electron Device Lett. 20161

[7] Ambacher O et al 2000 J. Appl. Phys. 87334 [8] Bernardini F, Fiorentini F and Vanderbilt D 1997 Phys. Rev. B 56 R10024

[9] Hsu L and Walukiewicz W 1997 Phys. Rev. B 561520

[10] Miyoshi M, Ishikawa H, Egawa T and Jimbo T 2003 Phys. Status Solidi c 02091

[11] Cordier Y, Hugues M, Lorenzini P, Semond F, Natali F and Massies J 2005 Phys. Status Solidi c 22720

[12] Ridley B K, Foutz B E and Eastman L F 2000 Phys. Rev. B 61 16862

[13] Dziuba Z, Antoszewski J, Dell J M, Farone L, Kozodoy P, Keller S, Keller B, Denbaars S P and Mishra U K 1997 J. Appl. Phys. 822996

[14] Antoszewski J and Faraone L 2004 Opto-Electron. Rev. 12347

[15] Vurgaftman I, Meyer J R, Hoffman C A, Redfern D, Antoszewski J, Farone L and Lindemuth J R 1998 J. Appl. Phys. 844966

[16] Antoszewski J, Faraone L, Vurgaftman I, Meyer J R and Hoffman C A 2004 J. Electron. Mater. 33673

[17] Swartz C H, Tomkins R P, Giles N C, Myers T H, Lu H, Schaff W J and Eastman L F 2004 J. Cryst. Growth 26929

[18] Swartz C H, Tomkins R P, Myers T H, Look D C and Sizelove J R 2005 J. Electron. Mater. 33412

[19] Biyikli N, Xie J, Moon Y-T, Yun F, Stefanita C-G, Bandyopadhyay S, Morkoc, H, Vurgaftman I and Meyer J R 2006 Appl. Phys. Lett. 88142106

[20] Zanato D, Gokden S, Balkan N, Ridley B K and Schaff W J 2004 Semicond. Sci. Technol. 19427

[21] Ridley B K 1982 J. Phys. C: Solid State Phys. 155899

[22] Ridley B K, Foutz B E and Eastman L F 1999 Phys. Rev. B 6116862

[23] Fang F F and Howard W E 1966 Phys. Rev. Lett. 16797 [24] Davies J H 1998 The Physics of Low-Dimensional Semiconductors (Cambridge: Cambridge University Press) [25] Ramonas M, Matulionis A and Rota L 2003 Semicond. Sci.

Technol. 18118

[26] Kearney M J and Horrell A I 1998 Semicond. Sci. Technol. 13 174

[27] Hess K 1979 Appl. Phys. Lett. 35484

[28] Lee K, Shur M S, Drummond T J and Morkoc H 1983 J. Appl.

Phys. 546432

[29] Fewster P F, Andrew N L and Curling C J 1988 J. Appl. Cryst. 21524

[30] Deveaud B, Emergy J Y, Chomette A, Lambert B and Baudet M 1985 Superlatt. Microstruct. 3205

[31] Hirakawa K and Sakaki H 1986 Phys. Rev. B 338291

[32] Gokden S 2003 Phys. Status Solidi a 2369 [33] Calle F, Palacios T, Monroy E, Grajal J, Verdu M, Bougrioua Z and Moerman I 2003 J. Mater. Sci.-Mater. El. 14271

[34] Ambacher O et al 1999 J. Appl. Phys. 853222

[35] Lisesivdin S B, Acar S and Kasap M 2007 Mat. Sci. Eng. B submitted

[36] Zhang G Y, Tong Y Z, Yang Z J, Jin S X, Li J and Gan Z Z 1997 Appl. Phys. Lett. 713376

[37] Gokden S, Ilgaz A, Balkan N and Mazzucato S 2004 Physica E 2586 
[38] Gaska R, Yang J W, Osinsky A, Chen Q, Khan M A, Orlov A

O, Snider G L and Shur M S 1998 Appl. Phys. Lett. 72707

[39] Huang D et al 2001 Solid State Electron. 45711

[40] Gokden S 2004 Physica E 23114 